\title{
DYNAMICS OF A CONTINUED FRACTION OF RAMANUJAN WITH RANDOM COEFFICIENTS
}

\author{
JONATHAN M. BORWEIN AND D. RUSSELL LUKE
}

Received 16 November 2004

We study a generalization of a continued fraction of Ramanujan with random, complexvalued coefficients. A study of the continued fraction is equivalent to an analysis of the convergence of certain stochastic difference equations and the stability of random dynamical systems. We determine the convergence properties of stochastic difference equations and so the divergence of their corresponding continued fractions.

\section{Introduction}

For the sequence of complex-valued random variables $a:=\left(a_{n}\right)_{n=1}^{\infty}$, with nonzero magnitude bounded above and below in probability, and the real parameter $b>1$, denote the continued fraction $\mathscr{Y}_{1}(a, b)$ by

$$
\mathscr{Y}_{1}(a, b):=\frac{1^{b} a_{1}^{2}}{1+\frac{2^{b} a_{2}^{2}}{1+\frac{3^{b} a_{3}^{2}}{1+\cdots}}}
$$

This is a generalization of a continued fraction studied by Ramanujan. The original continued fraction, which can be written as $\mathscr{S}_{1}(a, 2)$, where $a=\left(a_{1}, a_{2}, a_{1}, a_{2}, \ldots\right)$ for $a_{1}$ and $a_{2}$ positive and real, satisfies a remarkable AGM relation, in addition to having applications to elliptic-function theory. Our interest in $\mathscr{S}_{1}(a, b)$ has grown out of investigations into the extent to which the properties of the original Ramanujan continued fraction can be generalized $[2,3,4,5]$. Our present focus is to establish the following sufficient conditions on sequences of random complex parameters $a=\left(a_{n}\right)_{n=1}^{\infty}$ for the divergence of $\mathscr{Y}_{1}(a, b)$.

Theorem 1.1 (sufficient conditions for divergence). For $0<\epsilon<b$ and $b>1$, let $a:=\left(a_{n}\right)$ be a random sequence of complex, zero-mean, independent random variables satisfying

$$
\begin{aligned}
& 0 \neq \prod_{n=1}^{\infty}\left(1-\frac{1}{(2 n)^{2 b} a_{2 n}^{2}}\right)<\infty, \quad 0 \neq \lim _{n \rightarrow \infty} \frac{a_{2}}{a_{2 n}^{2 n-1} a_{2 n-1}^{2 n-2}} \prod_{j=1}^{2 n-2} a_{j}^{2}<\infty \quad \text { a.s. }, \\
& \sum_{n}^{\infty} \frac{1}{n^{2(b-\epsilon)}} \operatorname{var}\left(\frac{1}{a_{2}} \prod_{j=1}^{n} \frac{a_{2 j-1}^{2}}{a_{2 j}^{2}}\right)<\infty, \quad \sum_{n}^{\infty} \frac{1}{n^{2(b-\epsilon)}} \operatorname{var}\left(\frac{a_{2}}{a_{2 n}^{2}} \prod_{j=1}^{n} \frac{a_{2 j}^{2}}{a_{2 j-1}^{2}}\right)<\infty .
\end{aligned}
$$


Then the stochastic Ramanujan continued fraction $\mathscr{S}_{1}(a, b)$ defined by (1.1) diverges almost surely with the even/odd parts of $\mathscr{Y}_{1}(a, b)$ converging almost surely to separate limits.

For our analysis, $\mathscr{S}_{1}(a, b)$, for $b>1$, is a straightforward generalization of the special case $\mathscr{Y}_{1}(a, 2)$, which we will denote by either $\mathscr{S}_{1}(a)$ or simply $\mathscr{Y}_{1}$ as follows:

$$
\mathscr{Y}_{1}(a)=\frac{1^{2} a_{1}^{2}}{1+\frac{2^{2} a_{2}^{2}}{1+\frac{3^{2} a_{3}^{2}}{1+\cdots}}} .
$$

We therefore restrict our attention to this particular continued fraction until the end of this paper.

To evaluate $\mathscr{S}_{1}$, we study the recurrence for the classical convergents $p_{n} / q_{n}$ to the fraction $\mathscr{Y}_{1}$. For a general continued fraction of the form

$$
\mathscr{S}_{\eta}(\gamma)=\eta_{0}+\frac{\gamma_{1}}{\eta_{1}+\frac{\gamma_{2}}{\eta_{2}+\frac{\gamma_{3}}{\eta_{3}+\cdots}}}
$$

these are defined by the truncated continued fraction: $p_{-1}=1, p_{0}=\eta_{0}, q_{-1}=0, q_{0}=1$, and

$$
\begin{aligned}
\mathscr{S}_{\eta}(\gamma) & \approx \frac{p_{1}}{q_{1}}=\frac{\eta_{1} p_{0}+\gamma_{1} p_{1}}{\eta_{1} q_{0}}=\eta_{0}+\frac{\gamma_{1}}{\eta_{1}} \quad \text { first order, } \\
& \approx \frac{p_{2}}{q_{2}}=\frac{\eta_{2} p_{1}+\gamma_{2} p_{0}}{\eta_{2} q_{1}+\gamma_{2} q_{0}}=\eta_{0}+\frac{\gamma_{1}}{\eta_{1}+\frac{\gamma_{1}}{\eta_{2} / \gamma_{2}}} \quad \text { second order, } \\
& \approx \cdots \\
& \approx \frac{p_{n}}{q_{n}}=\frac{\eta_{n} p_{n-1}+\gamma_{n} p_{n-2}}{\eta_{n} q_{n-1}+\gamma_{n} q_{n-2}}=\eta_{0}+\frac{\gamma_{1}}{\eta_{1}+\frac{\gamma_{1}}{\eta_{2}}} \quad n \text {th order. }
\end{aligned}
$$

A simple induction argument establishes the general recurrence for the numerator and denominator $p_{n}$ and $q_{n}$ shown above, namely,

$$
p_{n}=\eta_{n} p_{n-1}+\gamma_{n} p_{n-2}, \quad q_{n}=\eta_{n} q_{n-1}+\gamma_{n} q_{n-2} .
$$

For the continued fraction $\mathscr{S}_{1}(a)$, we have

$$
q_{n}=q_{n-1}+n^{2} \alpha_{n} q_{n-2}, \quad \alpha_{n}:=a_{n}^{2} .
$$


We will use $\alpha_{n}$ and $a_{n}^{2}$ interchangeably throughout. The $p_{n}$ terms of the classical convergents also satisfy (1.7).

Following $[2,5]$, it is helpful to consider the renormalized sequence $\left(v_{n}\right)$, where

$$
v_{n}:=\frac{q_{n}}{\Gamma(n+3 / 2) a_{n}^{n+1}} .
$$

A standard identity $[8$, equation $(1.2 .10)]$ for the separation of the convergents to $\mathscr{I}_{1}$ yields

$$
\frac{p_{n}}{q_{n}}-\frac{p_{n-1}}{q_{n-1}}=\frac{(-1)^{n-1} n !^{2}}{q_{n} q_{n-1}} \prod_{j=1}^{n} \alpha_{j} .
$$

In terms of the renormalized sequence $\left(v_{n}\right)$, this is

$$
\frac{p_{n}}{q_{n}}-\frac{p_{n-1}}{q_{n-1}}=\frac{(-1)^{n-1}}{v_{n} v_{n-1} a_{n}^{n+1} a_{n-1}^{n}}\left(\prod_{j=1}^{n} \alpha_{j}\right)\left\{1+O\left(\frac{1}{n}\right)\right\} .
$$

From the above preliminary analysis, it is immediately clear that for $\left|a_{n}\right|=\left|a_{m}\right| \neq 0$ for all $n, m \in \mathbb{N}$, the continued fraction $\mathscr{S}_{1}$ diverges - that is, the convergents separate-if

$$
\left(v_{n}\right) \text { is bounded. }
$$

The case of cyclic and arbitrary deterministic sequences of parameters $a_{n}$ has been treated in [5]. To tie the present theory to more classical results, we briefly discuss the case of complex parameters with constant phase in Section 3. In Section 4, we broaden our scope to general random sequences. Before proceeding with the analysis, however, we motivate this study in Section 2 with some numerical experiments of specific examples.

\section{Numerical motivation}

For different cases of the parameters $a_{n}$ in the continued fraction $\mathscr{S}_{1}$, we plot in the complex plane odd and even iterates of the recurrence

$$
v_{n}=\frac{2}{a_{n}(2 n+1)}\left(\frac{a_{n-1}}{a_{n}}\right)^{n} v_{n-1}+\frac{4 n^{2}}{(2 n-1)(2 n+1)}\left(\frac{a_{n-2}}{a_{n}}\right)^{n-1} v_{n-2},
$$

which follows directly from the rescaling (1.8). Our examples focus on the case $\left|a_{n}\right|=b$ for all $n$, and, in particular (without loss of generality) $\left|a_{n}\right|=1$. As a point of reference, we reproduce in Figures 2.1-2.2 the dynamics for periodic $\left(a_{n}\right)$ with cycle length 1, 2, 3, and 4 , and each $a_{n}$ being a root of unity. These cases have been studied at length in [5]. It appears from these simulations that the sequence $\left(v_{n}\right)$ is bounded for even-length cycles, hence $\mathscr{S}_{1}$ diverges. This has been confirmed in [5] for these parameter values. Odd-length cycles display a richer variety of behaviors, not all convergent, as shown in Figures 2.2(b)$2.2(\mathrm{c})$. 


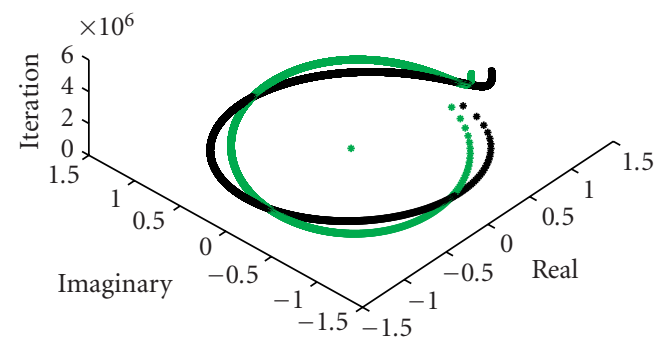

(a)

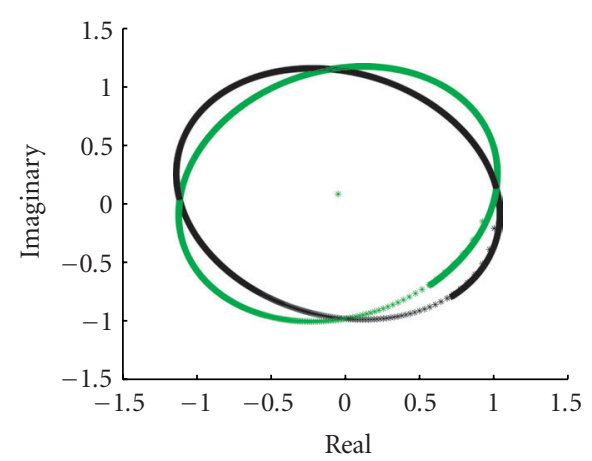

(b)

Figure 2.1. Dynamics for cycles of length $c=1$. Shown are the iterates $v_{n}$ given by (2.1) with $a_{n}=$ $\exp (i \pi / 2)$ for all $n$. Odd iterates are light, even iterates are dark.

A remarkable fact is that, even if the sequences $\left(a_{n}\right)$ are chosen to have constant magnitude and random phase, that is, for $a_{n}=c e^{i \theta_{n}}$ with $\theta_{n}$ randomly distributed on $[0,2 * \pi]$, the odd and even iterates $v_{n}$ demonstrate a surprising amount of structure. This is shown in Figure 2.3. We explain this remarkable regularity in the following sections.

\section{Random modulus/fixed phase}

We consider parameters $a_{n}$ of $\mathscr{Y}_{1}$ of the form

$$
a_{n}=c_{n} e^{i \theta}, \quad c_{n} \in \mathbb{R} \forall n, \theta \text { fixed. }
$$

The following result is an appplication of Stieltjes' convergence theorem for continued fractions $[7,8]$, and was pointed out to us by an anonymous referee.

Theorem 3.1 (random modulus, fixed phase). The generalized Ramanujan continued fraction $\mathscr{S}_{1}$ converges whenever all parameters satisfy $a_{n}=\left|a_{n}\right| e^{i \theta}$, for $\theta \in[-\pi, \pi] \backslash\{-\pi / 2$, $\pi / 2\}$ fixed, and for $0<m \leq\left|a_{n}\right| \leq M<\infty$. 


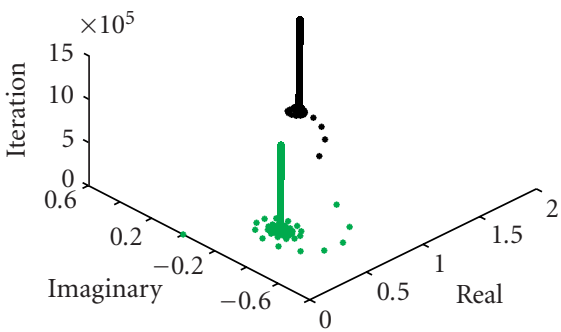

(a)

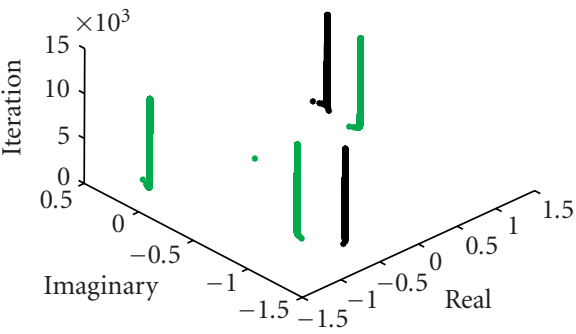

(c)

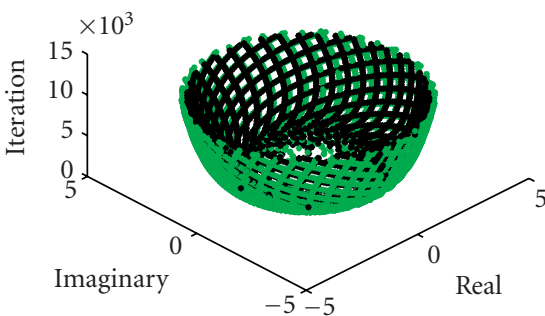

(b)

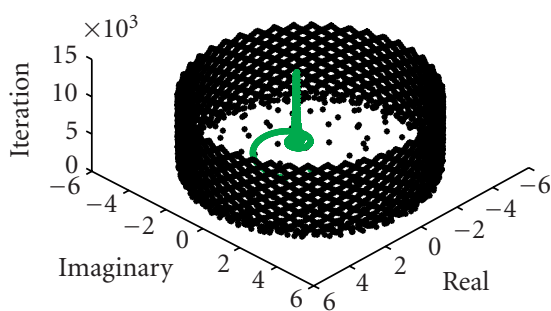

(d)

Figure 2.2. Dynamics for cycles of length $c=2,3$, and 4. Shown are the iterates $v_{n}$ given by $(2.1)$ with (a) $\left(a_{1}, a_{2}\right)=(\exp (i \pi / 4), \exp (i \pi / 6)),(\mathrm{b})\left(a_{1}, a_{2}, a_{3}\right)=(\exp (i \pi / 4), \exp (i \pi / 4), \exp (i(\pi / 4+1 / \sqrt{2}))$, (c) $\left(a_{1}, a_{2}, a_{3}\right)=(\exp (i \pi / 2), \exp (i \pi / 6), \exp (-i \pi / 6))$, and (d) $a_{1}=a_{3}=\exp (i \pi / 4), a_{2}=\exp (i \pi / 6), a_{4}=$ $\exp (i(\pi / 6+1 / 2))$. Odd iterates are light, even iterates are dark.

Proof. Write $\mathscr{Y}_{1}$ as a reduced continued fraction $\hat{\mathscr{S}}_{1}$ with coefficients $A_{i}>0$, that is,

$$
\hat{\mathscr{S}}_{1}(a)=\frac{1}{A_{1}+\frac{1}{A_{2}+\frac{1}{A_{3}+\cdots}}}
$$

where

$$
A_{n}= \begin{cases}\frac{n !^{2}}{(n / 2) !^{4}} 4^{-n} \prod_{j=1}^{n / 2} \frac{a_{2 j-1}^{2}}{a_{2 j}^{2}} & (n \text { even }), \\ \frac{((n-1) / 2) !^{4}}{n !^{2}} \frac{4^{n-1}}{a_{n}^{2}} \prod_{j=1}^{(n-1) / 2} \frac{a_{2 j}^{2}}{a_{2 j-1}^{2}} & (n \text { odd }) .\end{cases}
$$




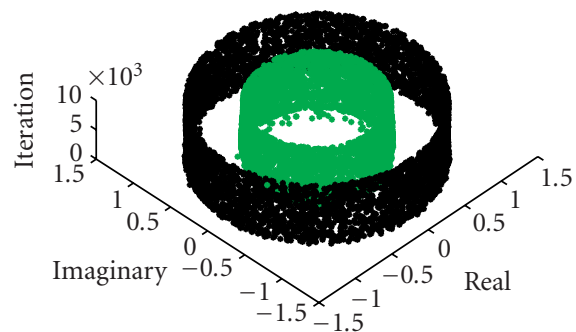

(a)

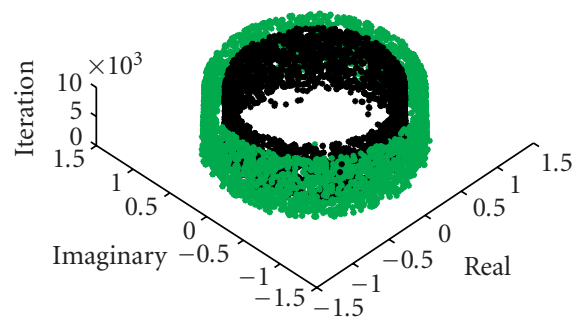

(c)

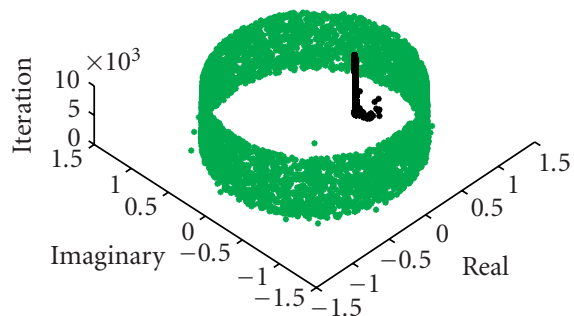

(b)

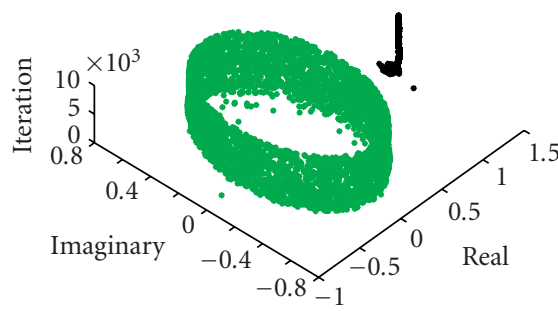

(d)

Figure 2.3. Dynamics for random cycles. Shown are the iterates $v_{n}$ given by (2.1) with (a) $a_{n}=$ $\exp \left(i \theta_{n}\right), \theta_{n} \sim U[0,2 \pi]$ (b) one random strand $\bmod 2, a_{2 n+1}=\exp (i \pi / 6), a_{2 n}=\exp \left(i \theta_{n}\right), \theta_{n} \sim$ $U[0,2 \pi],(\mathrm{c})$ one random strand $\bmod 3, a_{3 n+1}=a_{3 n+2}=\exp (i \pi / 6), a_{3 n}=\exp \left(i \theta_{n}\right), \theta_{n} \sim U[0,2 \pi]$, and (d) one random strand $\bmod 4, a_{4 n+1}=a_{4 n+2}=a_{4 n+3}=\exp (i \pi / 6), a_{4 n}=\exp \left(i \theta_{n}\right), \theta_{n} \sim U[0,2 \pi]$. Odd iterates are light, even iterates are dark.

The reduced continued fraction is of the form studied by Stieltjes

$$
\hat{\mathscr{S}}_{1}=\frac{1}{d_{1} z}+\frac{1}{d_{2}}+\frac{1}{d_{3} z}+\frac{1}{d_{4}}+\cdots
$$

with $z=e^{-2 \mathrm{i} \theta}$ and

$$
d_{n}= \begin{cases}\frac{n !^{2}}{(n / 2) !^{4}} 4^{-n} \prod_{j=1}^{n / 2} \frac{a_{2 j-1}^{2}}{a_{2 j}^{2}}=\left(\frac{2}{n \pi}+O\left(\frac{1}{n^{2}}\right)\right) \prod_{j=1}^{n / 2} \frac{a_{2 j-1}^{2}}{a_{2 j}^{2}} & (n \text { even }), \\ \frac{((n-1) / 2) !^{4}}{n !^{2}} 4^{n-1} \prod_{j=1}^{(n-1) 2} \frac{a_{2 j}^{2}}{a_{2 j-1}^{2}}=\left(\frac{2}{n \pi}+O\left(\frac{1}{n^{2}}\right)\right) \prod_{j=1}^{(n-1) / 2} \frac{a_{2 j}^{2}}{a_{2 j-1}^{2}} & \text { ( } n \text { odd }) .\end{cases}
$$

Note that for $a_{n}=\left|a_{n}\right| e^{\mathrm{i} \theta}$ with $0<m \leq\left|a_{n}\right| \leq M<\infty$, the sum of the coefficients $d_{n}$ is unbounded. Convergence then follows from the Stieltjes convergence theorem for continued fractions [8], which asserts that $\hat{\mathscr{S}}_{1}$ converges at the point $z \in\{w \in \mathbb{C}|| \arg w \mid<\pi\}$ if and only if $\sum d_{n}=\infty$. 
Remark 3.2. The Stieltjes convergence theorem actually says much more, namely, that the continued fraction converges locally uniformly in $\mathscr{T}:=\{z \in \mathbb{C}|| \arg z \mid<\pi\}$ to a holomorphic function $f\left(e^{-2 i \theta}\right)$. But even, this is a special case of the more general parabola theorem [7].

\section{General random parameters}

In this section, we pursue a general theory for complex random parameters $a_{n}$ bounded above and below almost surely. Our principal tools draw from a matrix analysis of $\mathscr{S}_{1}$ based on the renormalized sequence $\left(v_{n}\right)$ defined by $(2.1)$. Though the phases of the parameters are entirely random, the sequence $\left(v_{n}\right)$ exhibits an odd/even behavior as the figures illustrate. To see why this might be, note that the recurrence is a 2-step backward difference equation. Reformulating (1.7) in terms of $2 \times 2$ matrices yields

$$
\mathbf{q}_{n}=Q_{n} \mathbf{q}_{n-1}, \quad Q_{n}:=\left[\begin{array}{cc}
1 & n^{2} \alpha_{n} \\
1 & 0
\end{array}\right], \mathbf{q}_{n}:=\left(\begin{array}{c}
q_{n} \\
q_{n-1}
\end{array}\right)
$$

The analogous sequence of vectors corresponding to the rescaling $v_{n}$ is

$$
\mathbf{v}_{n}:=\left(\begin{array}{c}
v_{n} \\
v_{n-1}
\end{array}\right)
$$

To show the odd/even behavior inherent in the difference equation, we examine the sequence

$$
\mathbf{v}_{n}=Y_{n} \mathbf{v}_{n-1}, \quad Y_{n}:=G_{n}^{-1} Q_{n} G_{n-1}
$$

for

$$
G_{n}:=\operatorname{Diag}\left(\Gamma\left(n+\frac{3}{2}\right) a_{n}^{n+1}, \Gamma\left(n+\frac{1}{2}\right) a_{n-1}^{n}\right) .
$$

Define the matrix $\hat{Y}_{n}$ by

$$
\hat{Y}_{n}:=Y_{2 n} Y_{2 n-1}
$$

This has the explicit representation

$$
\hat{Y}_{n}=\left[\begin{array}{ll}
\hat{y}_{1,1}^{(n)} & \hat{y}_{1,2}^{(n)} \\
\hat{y}_{2,1}^{(n)} & \hat{y}_{2,2}^{(n)}
\end{array}\right]
$$


where

$$
\begin{aligned}
& \hat{y}_{1,1}^{(n)}:=\left(\frac{\alpha_{2 n-2}}{\alpha_{2 n}}\right)^{n}\left(\frac{1}{\alpha_{2 n-2} \alpha_{2 n}}\right)^{1 / 2}\left(\frac{1+4 n^{2} \alpha_{2 n}}{4 n^{2}-1 / 4}\right), \\
& \hat{y}_{1,2}^{(n)}:=\left(\frac{\alpha_{2 n-3}}{\alpha_{2 n}}\right)^{n}\left(\frac{\alpha_{2 n-1}}{\alpha_{2 n-3} \alpha_{2 n}^{1 / 2}}\right)\left(\frac{(2 n-1)^{2}}{(2 n-3 / 2)\left(4 n^{2}-1 / 4\right)}\right), \\
& \hat{y}_{2,1}^{(n)}:=\left(\frac{\alpha_{2 n-2}}{\alpha_{2 n-1}}\right)^{n} \frac{1}{\alpha_{2 n-2}^{1 / 2}} \frac{1}{(2 n-1 / 2)} \\
& \hat{y}_{2,2}^{(n)}:=\left(\frac{\alpha_{2 n-3}}{\alpha_{2 n-1}}\right)^{n-1}\left(\frac{(2 n-1)^{2}}{(2 n-1)^{2}-1 / 4}\right) .
\end{aligned}
$$

The determinant of this general $\hat{Y}_{n}$ is

$$
\operatorname{det}\left(\hat{Y}_{n}\right)=\left(\frac{\alpha_{2 n-2}}{\alpha_{2 n}}\right)^{n-1 / 2}\left(\frac{\alpha_{2 n-3}}{\alpha_{2 n-1}}\right)^{n-1} \frac{64 n^{2}(2 n-1)^{2}}{(4 n-3)(4 n-1)^{2}(4 n+1)}
$$

The identity

$$
\prod_{n=2}^{\infty} \frac{64 n^{2}(2 n-1)^{2}}{(4 n-3)(4 n-1)^{2}(4 n+1)}=\frac{\pi}{2}
$$

follows readily from the Wallis/Stirling formula [1]. Hence,

$$
\lim _{n \rightarrow \infty} \operatorname{det}\left(\mathscr{Y}_{n}\right)=\frac{\pi}{2} \beta, \quad \mathscr{Y}_{n}=\prod_{j=1}^{n} \hat{Y}_{n}
$$

which, of course, depends on the existence of the limit

$$
\beta:=\prod_{n=2}^{\infty}\left(\frac{\alpha_{2 n-2}}{\alpha_{2 n}}\right)^{n-1 / 2}\left(\frac{\alpha_{2 n-3}}{\alpha_{2 n-1}}\right)^{n-1}=\lim _{n \rightarrow \infty} \frac{\alpha_{2}^{1 / 2}}{\alpha_{2 n}^{n-1 / 2} \alpha_{2 n-1}^{n-1}} \prod_{j=1}^{2 n-2} \alpha_{j} .
$$

Convergence of the determinant, however, is no guarantee of the same for the matrices $\mathscr{Y}_{n}$. Proving that the matrices converge is the object of the analysis that follows.

For the moment, assume that $\lim _{n \rightarrow \infty} \mathscr{Y}_{n}=y^{\infty}$, where $y^{\infty}$ is a finite complex random matrix. We then have the following generalization of [2, Theorem 4.1] concerning the convergence of odd and even parts of $\mathscr{Y}_{1}(a)$.

Theorem 4.1 (odd and even convergents of random continued fractions). Let the sequence of complex random variables $\left(a_{n}\right)_{n=1}^{\infty}$ satisfy

$$
0 \neq \beta:=\lim _{n \rightarrow \infty} \frac{a_{2}}{a_{2 n}^{2 n-1} a_{2 n-1}^{2 n-2}} \prod_{j=1}^{2 n-2} a_{j}^{2} \quad \text { a.s. }
$$

For the corresponding continued fraction $\mathscr{S}_{1}(a)$ defined by $(1.3)$, let $\left(u_{n}\right)$ be the analog to $\left(v_{n}\right)$ in (1.8) with $q_{n}$ replaced by $p_{n}$. If the matrix $\mathscr{Y}_{n}$ defined by (4.10) converges almost 
surely to the finite random matrix $y^{\infty}$, then for the standard initial conditions

$$
\left(u_{-1}, u_{0}, v_{-1}, v_{0}\right)=\left(\frac{1}{\sqrt{\pi}}, 0,0, \frac{2}{a_{0} \sqrt{\pi}}\right)
$$

the even and odd parts of $\mathscr{S}_{1}(a)$ are given by

$$
\mathscr{Y}_{1}^{\text {(even) }}(a)=\frac{a_{0} y_{1,2}^{\infty}}{2 y_{1,1}^{\infty}}, \quad \mathscr{Y}_{1}^{\text {(odd) }}(a)=\frac{a_{0} y_{2,2}^{\infty}}{2 y_{2,1}^{\infty}}
$$

where $y_{i, j}^{\infty}$ is the $i, j$ th element of $y^{\infty}$. These limits are almost surely not equal, thus $\mathscr{Y}_{1}$ diverges almost surely. Indeed, the separation of odd and even limits is given explicitly by

$$
\mathscr{Y}_{1}^{(\text {even })}(a)-\mathscr{Y}_{1}^{(\text {odd })}(a)=-\frac{a_{0}^{2} \pi}{4 a_{2} y_{1,1}^{\infty} y_{2,1}^{\infty}} \beta .
$$

Proof. The first relation (4.14) is immediate from the definition of the classical convergents. The limits cannot be equal since otherwise we would have

$$
\frac{a_{0} y_{1,2}^{\infty}}{2 y_{1,1}^{\infty}}=\frac{a_{0} y_{2,2}^{\infty}}{2 y_{2,1}^{\infty}} \Longrightarrow y_{1,1}^{\infty} y_{2,2}^{\infty}-y_{1,2}^{\infty} y_{2,1}^{\infty}=0
$$

whence, from (4.10), $\beta=0$. But this contradicts the assumption that $\beta \neq 0$ almost surely. To see (4.15), note that by (1.10) and the initial condition $\left(v_{-1}, v_{0}\right)=\left(0,2 /\left(a_{0} \sqrt{\pi}\right)\right)$,

$$
\begin{aligned}
\mathscr{Y}_{1}^{\text {(even) }}(a)-\mathscr{Y}_{1}^{\text {(odd })}(a) & =\lim _{n \rightarrow \infty}-\frac{\left(\prod_{j=1}^{2 n} \alpha_{j}\right)}{v_{2 n} v_{2 n-1} \alpha_{2 n}^{n+1 / 2} \alpha_{2 n-1}^{n}} \\
& =\lim _{n \rightarrow \infty}-\frac{a_{0}^{2} \pi}{4 y_{1,1}^{(n)} y_{2,1}^{(n)}} \frac{\left(\prod_{j=1}^{2 n} \alpha_{j}\right)}{\alpha_{2 n}^{n+1 / 2} \alpha_{2 n-1}^{n}}
\end{aligned}
$$

where $y_{i, j}^{(n)}$ is the $i, j$ th element of the matrix $\mathscr{Y}_{n}$ defined by (4.10). The limit above, together with (4.11), yields

$$
\mathscr{S}_{1}^{(\text {even })}(a)-\mathscr{Y}_{1}^{(\text {odd })}(a)=-\frac{a_{0}^{2} \pi}{4 a_{2} y_{1,1}^{\infty} y_{2,1}^{\infty}} \beta
$$

Remark 4.2. If $\beta=0$ with probability $>0$, then the analysis is indeterminate. Formally, from the definition of the classical convergents, we have

$$
\frac{2}{a_{0} \sqrt{\pi}} y_{1,1}^{\infty} \mathscr{J}_{1}^{(\mathrm{even})}=\frac{1}{\sqrt{\pi}} y_{1,2}^{\infty}, \quad \frac{2}{a_{0} \sqrt{\pi}} y_{2,1}^{\infty} \varphi_{1}^{(\text {odd })}=\frac{1}{\sqrt{\pi}} y_{2,2}^{\infty} .
$$

Multiplying the equation on the left by $y_{2,1}^{\infty}$ and the right by $y_{1,1}^{\infty}$ and then subtracting yield

$$
\frac{1}{\sqrt{\pi}}\left(y_{1,1}^{\infty} y_{2,2}^{\infty}-y_{1,2}^{\infty} y_{2,1}^{\infty}\right)=\frac{2}{a_{0} \sqrt{\pi}} y_{2,1}^{\infty} y_{1,1}^{\infty}\left(\mathscr{Y}_{1}^{(\text {even })}-\mathscr{Y}_{1}^{(\text {odd })}\right)
$$


But if $\beta=0$, by (4.10) we have $y_{1,1}^{\infty} y_{2,2}^{\infty}-y_{1,2}^{\infty} y_{2,1}^{\infty}=0$, and so $y_{2,1}^{\infty} y_{1,1}^{\infty}\left(\varphi_{1}^{\text {(even) }}-\varphi_{1}^{\text {(odd) })}\right)=$ 0 . We cannot determine from this analysis whether the separation of the odd and even convergents is zero as would be the case if $\mathscr{S}_{1}$ were to converge.

What remains, then, is to determine the conditions under which $\mathscr{Y}_{n}$ converges as $n \rightarrow$ $\infty$. To begin, we extract the leading-order behavior. Since the coefficients $a_{n}$ are nonzero with magnitude bounded above and below in probability, for each $n$, the elements $\hat{y}_{i j}^{(n)}$ of $\hat{Y}_{n}$ are almost surely bounded, so we can expand $\hat{Y}_{n}$ in powers of $n^{-1}$ to get

$$
\hat{Y}_{n}=K_{n}+\frac{1}{2 n} W_{n}+O\left(n^{-2}\right)
$$

where

$$
\begin{aligned}
K_{n} & =\left[\begin{array}{cc}
\left(\frac{\alpha_{2 n-2}}{\alpha_{2 n}}\right)^{n-1 / 2} & 0 \\
0 & \left(\frac{\alpha_{2 n-3}}{\alpha_{2 n-1}}\right)^{n-1}
\end{array}\right], \\
W_{n} & =\left[\begin{array}{cc}
0 & \frac{1}{\alpha_{2 n}^{1 / 2}}\left(\frac{\alpha_{2 n-3}}{\alpha_{2 n}}\right)^{n}\left(\frac{\alpha_{2 n-1}}{\alpha_{2 n-3}}\right) \\
\frac{1}{\alpha_{2 n-2}^{1 / 2}}\left(\frac{\alpha_{2 n-1}}{\alpha_{2 n-2}}\right)^{-n} & 0
\end{array}\right] .
\end{aligned}
$$

Hence,

$$
\mathscr{y}_{n}=\prod_{j=2}^{n}\left(K_{j}+\frac{1}{2 j} W_{j}+O\left(j^{-2}\right)\right)=u_{n}+O\left(n^{-2}\right)
$$

where

$$
u_{n}:=\prod_{j=2}^{n} K_{j}+\frac{1}{2 j} W_{j}
$$

By induction on $n$, this factors as

$$
u_{n}=\left(\prod_{j=2}^{n} K_{j}\right) \prod_{j=2}^{n}\left(I+\frac{1}{2 j} \widehat{W}_{j}\right)
$$

where

$$
\widehat{W}_{n}:=\left(\prod_{j=2}^{n} K_{j}\right)^{-1}\left(\prod_{j=2}^{n-1} \widehat{K}_{j}\right) W_{n}
$$

for

$$
\hat{K}_{j}=\left[\begin{array}{ll}
0 & 1 \\
1 & 0
\end{array}\right] K_{j}\left[\begin{array}{ll}
0 & 1 \\
1 & 0
\end{array}\right]
$$


As it turns out, $\widehat{W}_{n}$ has a simple explicit representation

$$
\widehat{W}_{n}=\frac{1}{a_{2 n}}\left[\begin{array}{cc}
0 & \omega_{n} \\
\omega_{n}^{-1} & 0
\end{array}\right], \quad \omega_{n}=\frac{a_{2 n}}{a_{2}} \prod_{j=1}^{n} \frac{\alpha_{2 j-1}}{\alpha_{2 j}} .
$$

To ease the computations, we focus our attention on the rotated product

$$
\hat{U}_{n}:=\left(\prod_{j=2}^{n} K_{j}\right)^{-1} u_{n}=\prod_{j=2}^{n}\left(I+\frac{1}{2 j} \widehat{W}_{j}\right) .
$$

The justification for this follows next.

THEOREM 4.3. If $\hat{U}_{n} \stackrel{\text { a.s. }}{\rightarrow} \hat{U}_{\infty}$ and $\prod_{j=2}^{n} K_{j} \stackrel{\text { a.s. }}{\rightarrow} \mathscr{K}_{\infty}$, where both $\hat{U}_{\infty}$ and $\mathscr{K}_{\infty}$ are nonsingular, then $\mathcal{U}_{n} \stackrel{\text { a.s. }}{\rightarrow} \mathcal{Y}_{\infty}^{-1} \widehat{U}_{\infty}$ and $\mathcal{Y}_{n} \stackrel{\text { a.s. }}{\rightarrow} \mathrm{Y}^{\infty}$, a finite random matrix.

Proof. This follows from (4.24) and [5, Theorem 5.3].

Theorem 4.3, together with Theorem 4.1, yields particularly clean sufficient conditions for the divergence of $\mathscr{Y}_{1}$, but note that we have pushed the question of convergence of $y_{n}$ onto the convergence of $\hat{U}_{n}$. We focus next on $\hat{U}_{n}$.

Remark 4.4 (parameter qualifications). We briefly summarize our strategy and the accompanying restrictions. The leading-order behavior of the matrix expansions in powers of $n^{-1}$ is guaranteed by the boundedness assumptions, above and below, on the parameters $a_{n}$ with which we began this work. Subsequent restrictions on the parameters have been added as we extract the critical behavior of the matrix products describing the dynamics of the related difference equation. The most severe restrictions on the sequences $\left(a_{n}\right)$ come from the invertibility assumption in Theorem 4.1 and that of $\hat{U}_{\infty}$ and $\mathscr{K}_{\infty}$ in Theorem 4.3. The first of these, that $\beta \neq 0$ where $\beta$ is defined by (4.11), was discussed in Remark 4.2. The assumption that $\prod_{j=1}^{n} K_{j} \stackrel{a . s .}{\rightarrow} \mathscr{K}_{\infty}$ invertible is equivalent to the condition

$$
0 \neq \lim _{n \rightarrow \infty} \operatorname{det}\left(\prod_{j=2}^{n} K_{j}\right)<\infty \quad \text { a.s. }
$$

which amounts to

$$
0 \neq \lim _{n \rightarrow \infty} \frac{\alpha_{2}^{1 / 2}}{\alpha_{2 n}^{n-1 / 2} \alpha_{2 n-1}^{n-1}} \prod_{j=1}^{2 n-2} \alpha_{j}<\infty \quad \text { a.s. }
$$

From (4.29), the remaining invertibility assumption, namely that $\hat{U}_{n} \stackrel{\text { a.s. }}{\rightarrow} \hat{U}_{\infty}$ invertible, is equivalent to the condition

$$
0 \neq \operatorname{det} \prod_{j=2}^{\infty}\left(I+\frac{1}{2 j a_{2 j}}\left[\begin{array}{cc}
0 & \omega_{n} \\
\omega_{n}^{-1} & 0
\end{array}\right]\right)<\infty \quad \text { a.s. }
$$


or, more simply,

$$
0 \neq \prod_{j=2}^{\infty}\left(1-\frac{1}{\left(2 j a_{2 j}\right)^{2}}\right)<\infty \quad \text { a.s. }
$$

Conditions (4.32) and (4.34) are fundamental to our analysis.

4.1. Stochastic matrix analysis. In this section, we will prove the following theorem. Theorem 4.5 (stochastic matrix products). Let

$$
\epsilon, b>0, \quad b-\epsilon>0, \quad b+\epsilon>1,
$$

and let $\left(\zeta_{j}\right)$ and $\left(\zeta_{j}^{\prime}\right)$ be sequences of zero-mean independent random variables that satisfy

$$
\sum_{j=1}^{\infty} \frac{1}{j^{2(b-\epsilon)}} \operatorname{var}\left(\zeta_{j}\right)<\infty, \quad \sum_{j=1}^{\infty} \frac{1}{j^{2(b-\epsilon)}} \operatorname{var}\left(\zeta_{j}^{\prime}\right)<\infty .
$$

Then the matrix product

$$
\prod_{j=1}^{n}\left(I+\frac{1}{(2 j)^{b}}\left[\begin{array}{cc}
0 & \zeta_{j} \\
\zeta_{j}^{\prime} & 0
\end{array}\right]\right)
$$

converges almost surely to a finite matrix as $n \rightarrow \infty$. If, in addition,

$$
0<\left|\prod_{j=1}^{\infty}\left(1-\frac{\zeta_{j}^{\prime} \zeta_{j}}{(2 j)^{2 b}}\right)\right|<\infty \quad \text { a.s. }
$$

then the matrix product converges almost surely to an invertible matrix.

To begin, we collect some useful facts about the rate of convergence of sequences. We denote the limit of the sequence of random variables $\left(a_{n}\right)$ by $a_{\infty}$, and denote by $\left(a_{n}\right) \prec$ $\left(\epsilon_{n}\right)$ almost sure convergence of $\left(a_{n}\right)$ when this is provided by $\left|a_{n}-a_{\infty}\right|=O\left(\epsilon_{n}\right)$ almost surely.

Lemma 4.6. Let $\left(a_{n}\right)$ and $\left(b_{n}\right)$ be complex sequences, let $\left(\epsilon_{n}\right)$ be a positive sequence, and let $\left(z_{n}\right)$ with $\left|z_{n}\right|=z \in \mathbb{R}_{+}$for all $n=1,2, \ldots$ be any complex number. Suppose that

$$
\left(a_{n}\right) \prec\left(\epsilon_{n}\right), \quad\left(b_{n}\right) \prec\left(\epsilon_{n}\right),
$$

then

$$
\left(a_{n}+b_{n}\right) \prec\left(\epsilon_{n}\right), \quad\left(a_{n} b_{n}\right) \prec\left(\epsilon_{n}\right), \quad\left(z_{n} a_{n}\right) \prec\left(\epsilon_{n}\right) .
$$

Proof. The first two relations are clear. The last relation follows immediately from $\mid z_{n} a_{n}-$ $z_{n} a_{\infty}|=z| a_{n}-a_{\infty} \mid$. 
We state next a fundamental result which will yield, eventually, the conditions (4.36) for convergence of infinite products of random variables. Recall that a sequence $\left(S_{n}\right)$ is a martingale with respect to the random sequence $\left(\zeta_{n}\right)$ if, for all $n \geq 1$,

(a)

$$
\mathscr{E}\left(S_{n}\right)<\infty
$$

(b)

$$
\mathscr{E}\left(S_{n+1} \mid \zeta_{1}, \ldots, \zeta_{n}\right)=S_{n}
$$

where $\mathscr{E}$ denotes the expectation. For example, the sequence of partial sums of random complex-valued variables with fixed modulus and random phase uniformly distributed on $[0,2 \pi)$ is a martingale.

LEMMA 4.7 (martingale convergence theorem). Let $\left(\zeta_{n}\right)$ be a sequence of zero-mean random variables. Denote the corresponding martingale of partial sums by $\left(S_{n}\right)=\left(\sum_{j=1}^{n} \zeta_{j}\right)$. If

$$
\mathscr{E}\left(\left|S_{n}\right|^{2}\right)<\infty
$$

then $S_{n}$ converges almost surely to a finite random variable $S_{\infty}$.

For the proof of Lemma 4.7, see [6, Theorems 7.8.1 and 7.8.2]. We are now ready to state the main building block for the proof of Theorem 4.5 .

Proposition 4.8. Let $\zeta_{n}$ be zero-mean independent random variables satisfying

$$
\sum_{j}^{\infty} \frac{\operatorname{var}\left(\zeta_{j}\right)}{j^{2(b-\epsilon)}}<\infty \quad \text { for } \epsilon, b>0,0<b-\epsilon .
$$

Then,

$$
\sum_{j=1}^{n} \frac{\zeta_{j}}{j^{b-\epsilon}} \stackrel{\text { a.s. }}{\longrightarrow} \Sigma_{\infty} \quad \text { as } n \longrightarrow \infty
$$

where $\Sigma_{\infty}$ is a finite random variable. Moreover,

$$
\left(\sum_{j=1}^{n} \frac{\zeta_{j}}{j^{b}}\right) \prec\left(\frac{1}{j^{\epsilon}}\right)
$$

Proof. Equation (4.45) is a slight modification of [6, Exercise 7.8.2] and follows immediately from Lemma 4.7. To prove (4.46), note that by (4.45), we have

$$
\sup _{n}\left|\sum_{j=1}^{n} \frac{\zeta_{j}}{j^{b-\epsilon}}\right|<\infty \quad \text { a.s. }
$$


This, together with Abel's transformation [10, equation (I.2.1)]

$$
\sum_{j=m}^{n} \frac{1}{j^{\epsilon}} \frac{\zeta_{j}}{j^{b-\epsilon}}=\sum_{j=m}^{n-1}\left(\left(\sum_{k=m}^{j} \frac{\zeta_{k}}{k^{b-\epsilon}}\right)\left(\frac{1}{j^{\epsilon}}-\frac{1}{(j+1)^{\epsilon}}\right)\right)+\frac{1}{n^{\epsilon}} \sum_{j=m}^{n} \frac{\zeta_{j}}{j^{b-\epsilon}} \quad(m<n),
$$

yields

$$
\left|\sum_{j=m}^{n} \frac{\zeta_{j}}{j^{b}}\right| \leq \frac{1}{n^{\epsilon}} \sup _{k}\left|\sum_{j=m}^{k} \frac{\zeta_{j}}{j^{b-\epsilon}}\right|=O\left(\frac{1}{n^{\epsilon}}\right) \quad \text { a.s. }
$$

LEMmA 4.9 (product convergence). Let $\zeta_{j}$ be zero-mean independent random variables satisfying (4.44). Then for $b>1 / 2$, the product

$$
\prod_{j=1}^{n}\left(1+\frac{1}{j^{b}} \zeta_{j}\right)
$$

converges almost surely as $n \rightarrow \infty$.

Proof. Since the elements $\zeta_{j}$ have zero mean, by Proposition $4.8, \zeta_{j}$ satisfy

$$
\sum_{j=1}^{n} \frac{\zeta_{j}}{j^{b}} \stackrel{a . s .}{\longrightarrow} \Sigma_{\infty} \quad \text { as } n \longrightarrow \infty .
$$

Also, since $\zeta_{j}$ have zero mean and satisfy (4.44), we have

$$
\sum_{j}\left|\frac{\zeta_{j}}{j^{b}}\right|^{2}<\infty
$$

Thus, by general sufficient criteria for the convergence of infinite complex products $[9$, the Coriolis test], the product (4.50) converges almost surely.

We are now ready to proceed with the proof of the main result of this section.

Proof of Theorem 4.5. Our proof follows the same pattern as that of [2, Theorem 8.1]. We split the matrices in the infinite product into upper and lower triangular pieces and show that the resulting submatrices and their products converge. Let

$$
U:=\left[\begin{array}{ll}
0 & 1 \\
0 & 0
\end{array}\right], \quad L:=\left[\begin{array}{ll}
0 & 0 \\
1 & 0
\end{array}\right]
$$

We write

$$
\left(I+\frac{1}{(2 j)^{b}}\left[\begin{array}{cc}
0 & \zeta_{j} \\
\zeta_{j}^{\prime} & 0
\end{array}\right]\right)=\left(I+\frac{1}{(2 j)^{b}} \zeta_{j} U\right)\left(I+\frac{1}{(2 j)^{b}} \zeta_{j}^{\prime} L\right)-\frac{1}{(2 j)^{2 b}} \zeta_{j} \zeta_{j}^{\prime} U L,
$$

and define the partial product

$$
\Pi_{U L}^{n}=\prod_{j=1}^{n}\left(\left(I+\frac{1}{(2 j)^{b}} \zeta_{j} U\right)\left(I+\frac{1}{(2 j)^{b}} \zeta_{j}^{\prime} L\right)\right) .
$$


For $n \in \mathbb{N}$, let

$$
\begin{aligned}
\Pi_{U}^{n}:=\prod_{j=1}^{n}\left(I+\frac{1}{(2 j)^{b}} \zeta_{j} U\right), & \Pi_{L}^{n}:=\prod_{j=1}^{n}\left(I+\frac{1}{(2 j)^{b}} \zeta_{j}^{\prime} L\right), \\
\sum_{n}:=\sum_{j=1}^{n} \frac{1}{(2 j)^{b}} \zeta_{j}, & \sum_{n}^{\prime}:=\sum_{j=1}^{n} \frac{1}{(2 j)^{b}} \zeta_{j}^{\prime} .
\end{aligned}
$$

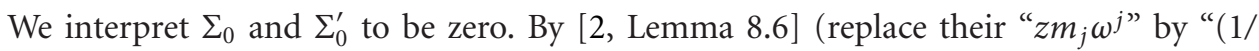
$(2 j)^{b} \zeta_{j}$ " and " $z m_{j} \omega^{-j "}$ by " $\left.\left(1 /(2 j)^{b}\right) \zeta_{j}^{\prime \prime}\right), \Pi_{U L}^{n}$ can be rewritten as

$$
\Pi_{U L}^{n}=\Pi_{U}^{n} \Pi_{L}^{n} \prod_{j=1}^{n}\left(I+R_{j}\right)
$$

where

$$
R_{n}:=\frac{1}{(2 n)^{b}} \zeta_{n}^{\prime}\left[\begin{array}{cc}
-\sum_{n-1}-\left(\Sigma_{n-1}\right)^{2} \Sigma_{n-1}^{\prime} & -\left(\Sigma_{n-1}\right)^{2} \\
\sum_{n-1} \Sigma_{n}^{\prime}+\sum_{n-1} \Sigma_{n-1}^{\prime}+\Sigma_{n-1}^{\prime} \Sigma_{n}^{\prime}\left(\Sigma_{n-1}\right)^{2} & \Sigma_{n-1}+\left(\Sigma_{n-1}\right)^{2} \Sigma_{n}^{\prime}
\end{array}\right] .
$$

By the definitions of $\Sigma_{0}$ and $\Sigma_{0}^{\prime}$, we have $R_{1}:=0$. The partial sums $\Sigma_{n}$ and $\Sigma_{n}^{\prime}$ converge almost surely by Proposition 4.8. By induction, it can be shown that

$$
\Pi_{U}^{n}=\left[\begin{array}{cc}
1 & \Sigma_{n} \\
0 & 1
\end{array}\right], \quad \Pi_{L}^{n}=\left[\begin{array}{cc}
1 & \Sigma_{n}^{\prime} \\
0 & 1
\end{array}\right]
$$

thus the sequences of matrices $\left(\Pi_{U}^{n}\right)$ and $\left(\Pi_{L}^{n}\right)$ converge almost surely. Hence, if $\prod_{j=1}^{n}(I+$ $R_{j}$ ) converges almost surely, then the sequence $\left(\Pi_{U L}^{n}\right)$ converges almost surely. We rewrite $R_{n}$ as

$$
R_{n}=\frac{\zeta_{n}^{\prime}}{(2 n)^{b}}\left[\begin{array}{ll}
r_{11}^{n} & r_{12}^{n} \\
r_{21}^{n} & r_{22}^{n}
\end{array}\right]
$$

where, by Lemma 4.6, (4.46), and (4.57),

$$
\left(r_{j k}^{n}\right) \prec\left(\frac{1}{n^{\epsilon}}\right) \quad \forall j, k
$$

Our strategy is to split $R_{n}$ into a sequence of absolutely convergent matrices and a convergent scaling of its limit. To this end, let

$$
R_{n}=P_{n}+\frac{\zeta_{n}^{\prime}}{(2 n)^{b}} T
$$

where

$$
P_{n}:=\frac{\zeta_{n}^{\prime}}{(2 n)^{b}}\left[\begin{array}{cc}
r_{11}^{n}-r_{11}^{\infty} & r_{12}^{n}-r_{12}^{\infty} \\
r_{21}^{n}-r_{21}^{\infty} & r_{22}^{n}-r_{22}^{\infty}
\end{array}\right], \quad T:=\left[\begin{array}{cc}
r_{11}^{\infty} & r_{12}^{\infty} \\
r_{21}^{\infty} & r_{22}^{\infty}
\end{array}\right]
$$


If

$$
\prod_{j=1}^{n}\left(I+\frac{\zeta_{j}^{\prime}}{(2 j)^{b}} T\right), \quad \sum_{j=1}^{\infty}\left|P_{n}\right|
$$

converge, then by [2, Theorem 6.1], the product $\prod_{j=1}^{n}\left(I+R_{j}\right)$ converges. Convergence of the product in (4.65) follows exactly as in the proof of [2, Lemma 8.7] and relies on Lemma 4.9. An examination of the eigenvalues of $P_{n}$ shows that, almost surely, $\left|P_{n}\right|=$ $O\left(1 / n^{b+\epsilon}\right)$. Thus, since by $(4.35) b+\epsilon>1$, we have that $\sum_{j=1}^{\infty}\left|P_{n}\right|$ converges almost surely.

Again, by Proposition 4.8, we have

$$
\sum_{j=1}^{n}\left|\frac{\zeta_{j} \zeta_{j}^{\prime}}{j^{2 b}}\right|<\infty
$$

whence convergence of the matrix sum

$$
\sum_{j=1}^{n}\left|\frac{\zeta_{j} \zeta_{j}^{\prime}}{j^{2 b}} U L\right|
$$

This proves the convergence of (4.37).

To complete the proof, note that

$$
\begin{aligned}
\operatorname{det} \hat{U}_{n} & =\operatorname{det} \prod_{j=1}^{n}\left(I+\frac{1}{(2 j)^{b}}\left[\begin{array}{cc}
0 & \zeta_{j} \\
\zeta_{j}^{\prime} & 0
\end{array}\right]\right) \\
& =\prod_{j=1}^{n} \operatorname{det}\left(I+\frac{1}{(2 j)^{b}}\left[\begin{array}{cc}
0 & \zeta_{j} \\
\zeta_{j}^{\prime} & 0
\end{array}\right]\right) \\
& =\prod_{j=1}^{n}\left(1-\frac{1}{(2 j)^{2 b}} \zeta_{j}^{\prime} \zeta_{j}\right) .
\end{aligned}
$$

This product is nonzero if $\left|1-\left(1 /(2 j)^{2 b}\right) \zeta_{j}^{\prime} \zeta_{j}\right| \geq m>0$ for all $j$, in which case $\hat{U}_{n}$ converges invertibly.

4.2. Application to continued fractions. Theorems 4.1, 4.3, and 4.5 together yield sufficient conditions for the divergence of the partial fraction $\mathscr{Y}_{1}$. We specialize these results to the case of continued fractions with parameters $\left(a_{n}\right)$ distributed uniformly on the unit circle in the complex plane as shown in Figure 2.3. Following Section 4.1, by (4.29), the random variables $\zeta_{n}$ and $\zeta_{n}^{\prime}$ in this case are given by

$$
\begin{gathered}
\zeta_{n}:=\frac{1}{a_{2 n}} \omega_{n}=\frac{1}{a_{2}}\left(\prod_{j=1}^{n} \frac{\alpha_{2 j-1}}{\alpha_{2 j}}\right), \\
\zeta_{n}^{\prime}:=\frac{1}{a_{2 n}} \omega_{n}^{-1}=\frac{a_{2}}{\alpha_{2 n}}\left(\prod_{j=1}^{n} \frac{\alpha_{2 j-1}}{\alpha_{2 j}}\right)^{-1} .
\end{gathered}
$$




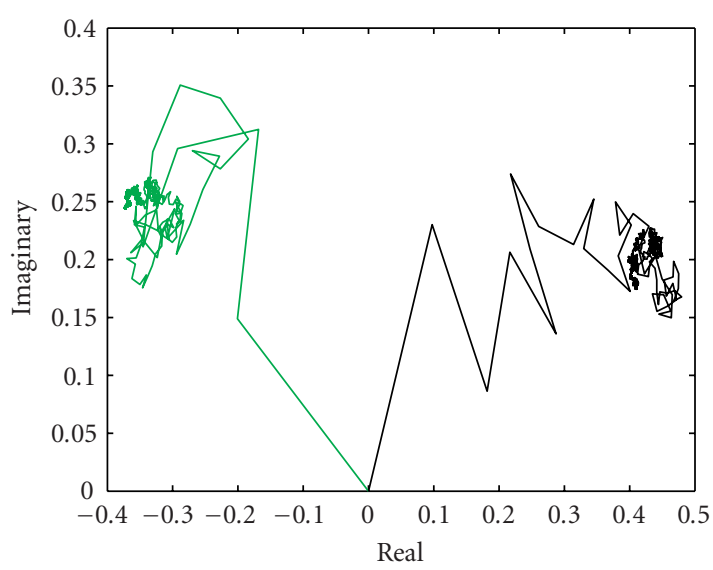

Figure 4.1. Random walks associated with parameter sequences of the form $\left(a_{n}\right)=\left(\exp \left(\mathrm{i}_{n}\right)\right)$ for $\theta_{n}$ a random variable uniformly distributed on $[0,2 \pi]$. The dark and light lines correspond to the random walks determined by the respective partial sums $\Sigma_{n}$ and $\Sigma_{n}^{\prime}$ defined in (4.72). The figure-an illustration of the convergence of the partial sums proven in Proposition 4.8-shows the location of the partial sum at step $n$ in the complex plane with lines connecting successive steps. The difference equation dynamics of the iterates $\left(v_{n}\right)$ defined by $(2.1)$ corresponding to these random walks are depicted in Figure 2.3a.

These are also random variables, uniformly distributed on the unit circle. By symmetry, it is immediate that $\mathscr{E}\left(\zeta_{n}\right)=\mathscr{E}\left(\zeta_{n}^{\prime}\right)=0$ and $\operatorname{var}\left(\zeta_{n}\right)$ and $\operatorname{var}\left(\zeta_{n}^{\prime}\right)$ are bounded for all $n$. Thus, for all $b-\epsilon>1 / 2$ with $1<\epsilon+b$ (in particular, for $b>3 / 4$ and $\epsilon$ small),

$$
\sum_{n} \frac{1}{n^{2(b-\epsilon)}} \operatorname{var}\left(\zeta_{n}\right)<\infty, \quad \sum_{n} \frac{1}{n^{2(b-\epsilon)}} \operatorname{var}\left(\zeta_{n}^{\prime}\right)<\infty .
$$

Define the partial sums by

$$
\Sigma_{n}:=\sum_{j}^{n} \frac{\zeta_{j}}{j^{b}}, \quad \Sigma_{n}^{\prime}:=\sum_{j}^{n} \frac{\zeta_{j}^{\prime}}{j^{b}}
$$

By Proposition 4.8, it follows that

$$
\lim _{n \rightarrow \infty} \sum_{n} \stackrel{\text { a.s. }}{\longrightarrow} \sum^{\infty}, \quad \lim _{n \rightarrow \infty} \sum_{n}^{\prime} \stackrel{\text { a.s. }}{\longrightarrow} \Sigma^{\infty \prime}
$$

Here $\Sigma^{\infty}$ and $\Sigma^{\infty \prime}$ are finite random variables. The partial sums represent a random walk. In Figure 4.1, we show one realization of this random walk with $b=1$ after $10^{4}$ steps. The continued fraction has $b=2$, so we have thus proved the divergence of $\mathscr{I}_{1}$ for the cases illustrated in Figure 2.3. By Theorem 4.1, the odd and even parts of $\mathscr{S}_{1}$ converge to separate limits as it is shown in the odd and even iterates of (2.1) converging to separate orbits. 


\section{Summary and open problems}

The analysis of Section 4.1, while specifically developed with continued fractions of the form $\mathscr{Y}_{1}(a)$ in mind, easily generalizes to continued fractions of the form $\mathscr{Y}_{1}(a, b)$ given by (1.1). It was shown in [2] that this leads to the rescaled sequence $\left(v_{n}^{(b)}\right)$, analogous to $(1.8)$

$$
v_{n}^{(b)}:=\frac{q_{n}}{\Gamma^{b / 2}(n+3 / 2) a_{n}^{n+1}} .
$$

The difference equation (2.1) then becomes

$$
v_{n}^{(b)}=\left(\frac{2}{2 n+1}\right)^{b / 2} \frac{1}{a_{n}}\left(\frac{a_{n-1}}{a_{n}}\right)^{n} v_{n-1}^{(b)}+\left(\frac{4}{(2 n-1)(2 n+1)}\right)^{b / 2} n^{2}\left(\frac{a_{n-2}}{a_{n}}\right)^{n-1} v_{n-2}^{(b)},
$$

and the matrix product

$$
u_{n}^{(b)}=\left(\prod_{j=2}^{n} K_{j}\right) \prod_{j=2}^{n}\left(I+\frac{1}{(2 j)^{b / 2}} \widehat{W}_{j}\right) .
$$

With this, we have established Theorem 1.1, in addition to sufficient conditions for the iterates $v_{n}^{(b)}$ to remain bounded. We recap with the following summary of our main results.

Theorem 5.1 (summary). For $\epsilon>0, b>1$, and $b-\epsilon>0$, let $a:=\left(a_{n}\right)$ be a random sequence of complex, zero-mean, independent random variables satisfying

$$
\begin{aligned}
& 0 \neq \prod_{n=1}^{\infty}\left(1-\frac{1}{(2 n)^{2 b} a_{2 n}^{2}}\right)<\infty, \quad 0 \neq \lim _{n \rightarrow \infty} \frac{a_{2}}{a_{2 n}^{2 n-1} a_{2 n-1}^{2 n-2}} \prod_{j=1}^{2 n-2} a_{j}^{2}<\infty \quad \text { a.s. }, \\
& \sum_{n}^{\infty} \frac{1}{n^{2(b-\epsilon)}} \operatorname{var}\left(\frac{1}{a_{2}} \prod_{j=1}^{n} \frac{a_{2 j-1}^{2}}{a_{2 j}^{2}}\right)<\infty, \quad \sum_{n}^{\infty} \frac{1}{n^{2(b-\epsilon)}} \operatorname{var}\left(\frac{a_{2}}{a_{2 n}^{2}} \prod_{j=1}^{n} \frac{a_{2 j}^{2}}{a_{2 j-1}^{2}}\right)<\infty .
\end{aligned}
$$

Then the iterates $v_{n}^{(b)}$ of the corresponding stochastic difference equation (5.2) are bounded almost surely and the stochastic Ramanujan continued fraction $\mathscr{S}_{1}(a, b)$ defined by $(1.1) \mathrm{di}$ verges almost surely with the even/odd parts of $\mathscr{S}_{1}(a, b)$ converging almost surely to separate limits.

Note that only sufficient conditions for divergence of random continued fractions have been determined with this analysis. The apparently more delicate question of necessary conditions for convergence remains open.

\section{Acknowledgments}

We thank an anonymous referee, Anja Sturm, Ian Coope, and Richard Crandall for helpful comments during the preparation of this work. Special thanks to Ray Mayer who laid the groundwork for the original and novel matrix theory in [2] that has proved so fruitful here. Russell Luke's work was supported in part by a Postdoctoral Fellowship from the Pacific Institute for the Mathematical Sciences at Simon Fraser University. 


\section{References}

[1] M. Abramowitz and I. A. Stegun, (eds.), Handbook of Mathematical Functions, 9th ed., Dover, New York, 1972.

[2] D. Borwein, J. M. Borwein, R. Crandall, and R. Mayer, On the dynamics of certain recurrence relations, to appear in Ramanujan J.

[3] J. M. Borwein and R. Crandall, On the Ramanujan AGM fraction, II: The complex-parameter case, Experiment. Math. 13 (2004), no. 3, 287-295.

[4] J. M. Borwein, R. Crandall, and G. Fee, On the Ramanujan AGM fraction, I: The real-parameter case, Experiment. Math. 13 (2004), no. 3, 275-285.

[5] J. M. Borwein and D. R. Luke, Dynamics of generalizations of the AGM continued fraction of Ramanujan. Part I: divergence, 2004, download: http://eprints.cecm.sfu.ca/archive/00000261.

[6] G. R. Grimmett and D. R. Stirzaker, Probability and Random Processes, Clarendon Press, Oxford University Press, New York, 1992.

[7] L. Lorentzen, A convergence question inspired by Stieltjes and by value sets in continued fraction theory, J. Comput. Appl. Math. 65 (1995), no. 1-3, 233-251.

[8] L. Lorentzen and H. Waadeland, Continued Fractions with Applications, Studies in Computational Mathematics, vol. 3, North-Holland, Amsterdam, 1992.

[9] E. M. E. Wermuth, Some elementary properties of infinite products, Amer. Math. Monthly 99 (1992), no. 6, 530-537.

[10] A. Zygmund, Trigonometric series, 2nd ed., vols. I, II, Cambridge University Press, New York, 1959.

Jonathan M. Borwein: Faculty of Computer Science, Dalhousie University, Halifax, NS, Canada B3H 1 W5

E-mail address: jborwein@cs.dal.ca

D. Russell Luke: Department of Mathematical Sciences, College of Arts and Sciences, University of Delaware, Newark, DE 19716-2553, USA

E-mail address: rluke@math.udel.edu 


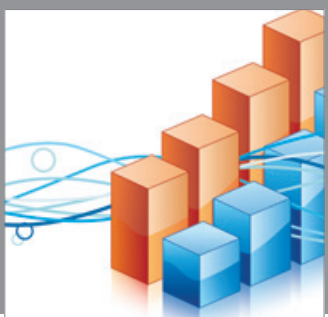

Advances in

Operations Research

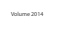

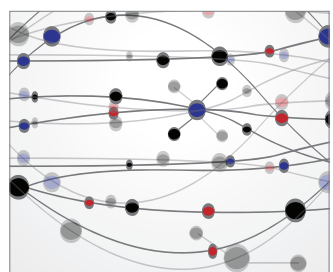

\section{The Scientific} World Journal
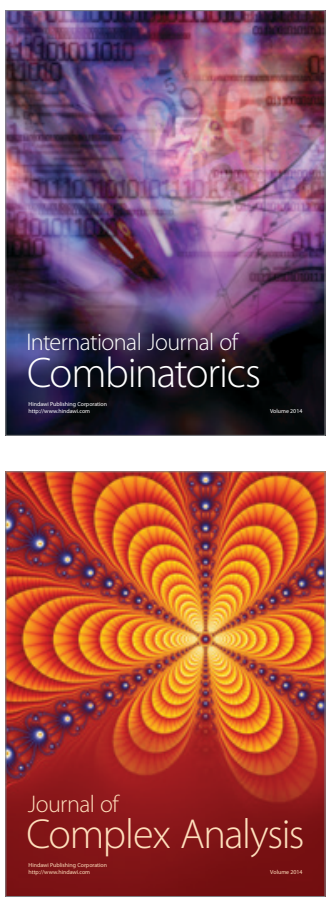

International Journal of

Mathematics and

Mathematical

Sciences
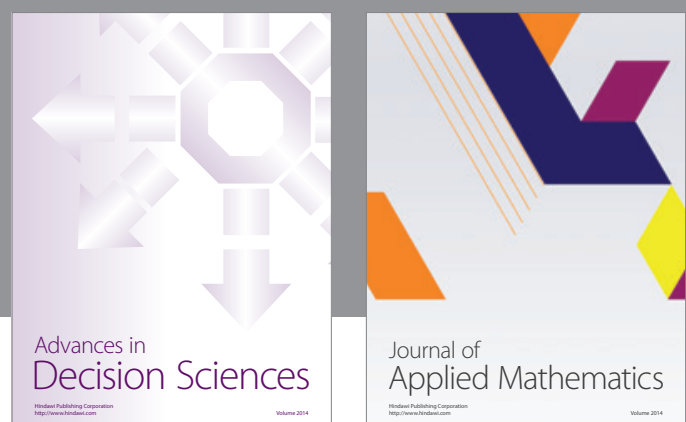

Journal of

Applied Mathematics
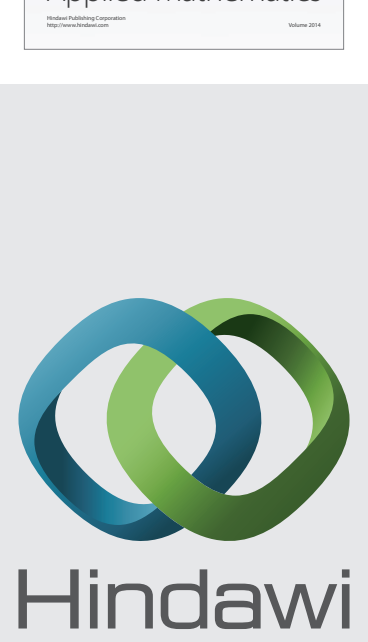

Submit your manuscripts at http://www.hindawi.com
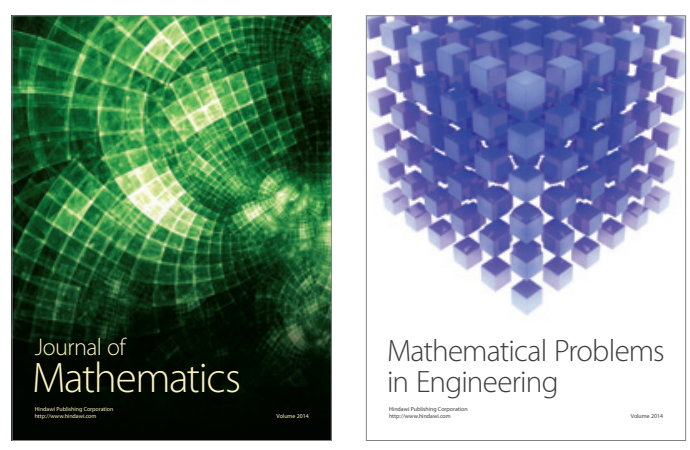

Mathematical Problems in Engineering
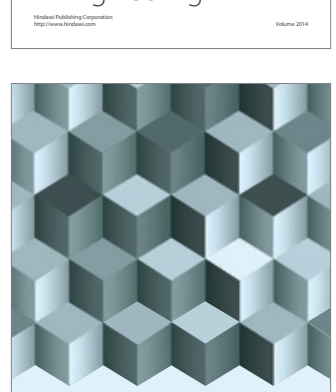

Journal of

Function Spaces
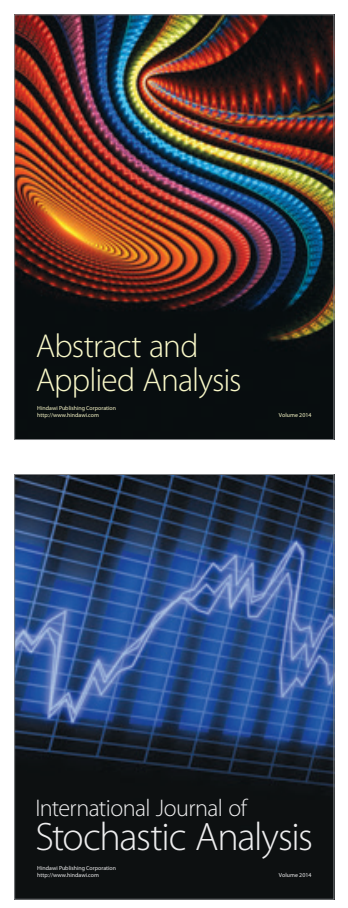

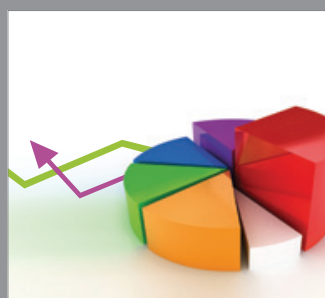

ournal of

Probability and Statistics

Promensencen
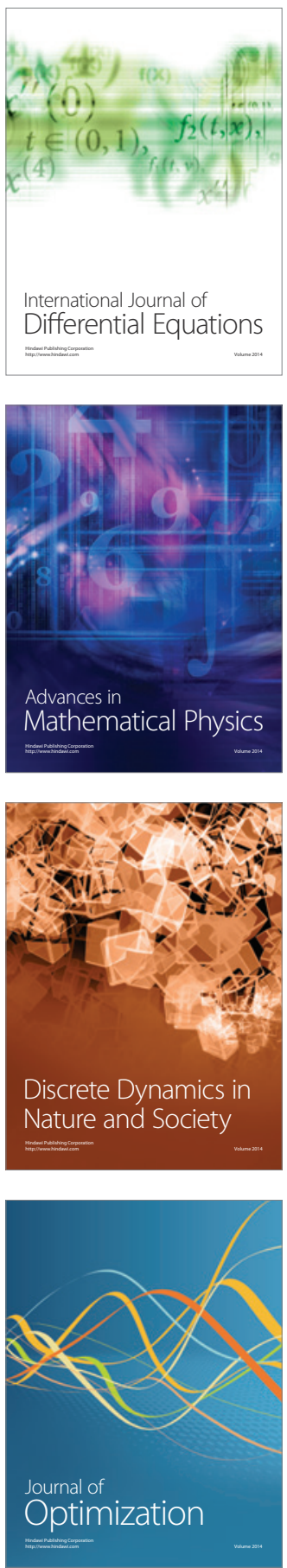